Clinical biochemistry

\title{
Interpretive comments on clinical biochemistry reports
}

\section{S Young}

\section{The need for quality assurance}

n the February issue of the journal, Sandle discussed current practice and future challenges in the management of external quality assurance in relation to clinical biochemistry, highlighting the need for an audit cycle of continuous improvement. ${ }^{1}$ One of the important areas touched upon was the quality assurance of interpretive comments on biochemistry reports; it was suggested that in the future individual participation in external quality assurance schemes of interpretive comments "may become a contractual rather than a professional obligation".

There is wide variation internationally and within the UK with regard to the extent to which individualised narrative interpretive comments are provided on biochemistry reports. Most biochemistry reports leave the laboratory without comment, or with only computer generated comments according to predetermined rules. However, the provision of individualised narrative comments on at least some biochemistry reports is part of routine practice in many laboratories. The objective is to aid clinicians in the interpretation of complex data, and comments will probably be of particular value when dynamic or uncommon tests are reported, where significant abnormalities are present, or where analytical or preanalytical factors (often not fully appreciated by the clinician) may impinge on the interpretation of the results. Several studies have described this interpretive process and indicated its value, ${ }^{2-4}$ although there is little evidence of improved patient outcomes.

The absence of common agreed international standards for working in this area has given rise to debate about the balance between the benefits and risks of providing written interpretive comments on biochemistry reports. In some laboratories, few if any individualised interpretive comments are provided, because of concern about the dangers of providing inappropriate advice in the absence of complete clinical information. A secondary concern is that providing such comments may delay the release of results by the laboratory to the detriment of patients, although results can be released when analytically validated, with the addition of individualised comments soon afterwards and before the release of a final report. In laboratories that choose not to provide written narrative comments, the interpretation of results is often still a crucial part of the laboratory service, but is provided at the bedside or by dialogue with clinicians. The advantage of this approach is that additional information to that provided on laboratory request forms may allow more accurate and appropriate interpretation of results.

"In some laboratories, few if any individualised interpretive comments are provided, because of concern about the dangers of providing inappropriate advice in the absence of complete clinical information"

External quality assurance of interpretive comments is at an early stage in its evolution, but schemes operate in several countries, including Australia and the UK. A recent report by Lim et al highlighted a high prevalence of less relevant and unacceptable comments by participants in the Australian scheme, including in a few cases comments that were considered dangerous. ${ }^{5}$ Similar findings were reported in the early stages of the comparable UK scheme. ${ }^{6}$ Lim et al suggested that the ability to comment should be restricted to individuals with clear expertise, although interestingly there was no evidence that official participants in the scheme (presumably with more expertise) performed better than unofficial participants. Errors were in some cases attributed to the absence of full clinical information, which is one of the dangers highlighted by those who choose not to provide written comments in routine practice.

Thus, what is the best way ahead with regard to narrative interpretive comments? First, evidence is required that the provision of individualised comments influences patient care or outcomes in a positive way, in view of the indications from Lim et al that in a considerable number of cases such comments may be misleading or even harmful. Although it is encouraging that users appear to like such comments, this is insufficient to justify their provision as a routine activity without knowledge that these comments are safe. Studies in this area will need to be carefully designed to compare the effects of providing written narrative comments with other approaches to providing interpretive advice to laboratory users, or with the provision of no advice except on request.

Second, quality assurance schemes for interpretive comments should be encouraged and developed. It is essential that those providing comments participate in such schemes, but because many clinical biochemists do not perform this activity in routine practice it would be foolish to make participation in such schemes a contractual or professional obligation for all.

J Clin Pathol 2005;58:575.

doi: 10.1136/jicp.2004.021055

Correspondence to: Professor I S Young, Department of Medicine, Wellcome Research Laboratories, Mulhouse Building, Royal Victoria Hospital, Grosvenor Road, Belfast BT12 6BJ,

UK; I.Young@qub.ac.uk

\section{REFERENCES}

1 Sandle LN. The management of external quality assurance: current practice and future challenges. J Clin Pathol 2005;58:141-4.

2 Dighe AS, Soderberg BL, Laposata M. Narrative interpretations for clinical laboratory interpretations. Am J Clin Pathol $2001 ; 116: 5123-8$.

3 Macmillan DH, Soderberg BL, Laposta M. Regulations regarding reflexive testing and narrative interpretations in laboratory medicine. Am J Clin Pathol 2001;116:S129-32.

4 Kratz A, Soderberg BL, Szczepiorkowski ZM, ef al. The generation of narrative interpretations in laboratory medicine. Am J Clin Pathol 2001:116:S133-40.

5 Lim EM, Sikaris KA, Gill J, et al. Quality assessment of interpretative commenting in clinical chemistry. Clin Chem 2004;50:632-7.

6 Marshall WJ, Challand GS. Provision of interpretative comments on biochemical repor forms. Ann Clin Biochem 2000;37:758-63. 